\title{
Hydrodynamization Time Near a Critical Point From a Holographic Bjorken Flow ${ }^{\dagger}$
}

\author{
Renato Critelli \\ Instituto de Física, Universidade de São Paulo, Rua do Matão, 1371 Butantã, CEP 05508-090 São Paulo, Brazil; \\ renato.critelli@usp.br \\ + Presented at the Hot Quarks 2018 - Workshop for Young Scientists on the Physics of Ultrarelativistic \\ Nucleus-Nucleus Collisions, Texel, The Netherlands, 7-14 September 2018.
}

Published: 13 May 2019

\begin{abstract}
This proceedings reviews recent progress in the study of far-from-equilibrium hydrodynamization process of strongly interacting matter in the vicinity of a critical point. From a full non-linear evolution of a gravitational theory dual to a conformal strongly coupled plasma, and starting from a non-equilibrium initial state, it is verified that the time it takes for the plasma to acquire hydrodynamic behavior greatly increases near the critical point.
\end{abstract}

Keywords: gauge/gravity duality; far-from-equilibrium dynamics; critical point

\section{Introduction}

Strongly coupled quantum plasmas are very challenging to study, either from an experimental or theoretical point of view. Although it is possible to study these systems in local equilibrium, it is sometimes also required to tackle the problem of far-from-equilibrium dynamics that represents the formation of the equilibrated plasma. This seems to be the case of the quark-gluon plasma (QGP) formed in ultrarelativistic heavy ion collisions [1,2], in which one expects that a non-equilibrium initial state quickly evolves in a nearly equilibrium state described by relativistic viscous hydrodynamics. Unfortunately, first principles calculations of this thermalization process in quantum chromodynamics (QCD) are not possible in lattice QCD[3] and, thus, one has to resort to effective models. In this context, the gauge/gravity duality [4] represents a powerful tool to investigate how thermalization occurs in strongly coupled plasmas [5].

Additionally, a better understanding of the QCD phase diagram in the $\left(T, \mu_{B}\right)$ plane, where $T$ is the temperature and $\mu_{B}$ is the baryon chemical potential [6], is needed. In this arena, lattice QCD has severe restrictions due to the sign problem [7] and can only successfully describe equilibrium properties of the QGP up to $\mu_{B} / T \sim 2$ [8]. With this limitation, one has to resort to effective models even for equilibrium phenomena and, once again, holography can be useful it easily incorporates chemical potential effects along with a critical point [9], which is long conjectured to exist in the QCD phase diagram.

The holographic AdS/CFT (anti-de Sitter/Conformal Field Theory) duality [4], which relates the type IIB string theory (quantum gravity) to the conformal $\mathcal{N}=4$ super Yang-Mills (SYM) in four dimensions, offers a unique opportunity to explore strongly coupled fluids since in the limit where the coupling of the CFT theory goes to infinity, the quantum gravity theory is well approximated by a classical theory of gravity in $\mathrm{AdS}_{5}$. In practical terms, this means that it is possible to study strongly coupled plasmas solving Einstein's equations of general relativity. Furthermore, the fact that holography naturally incorporates nearly perfect fluidity (i.e., $\eta / s=1 /(4 \pi)$, where $\eta$ is the shear viscosity and $s$ the entropy density, for a broad range of holographic theories [10]) of the QGP has sparked a lot of interest in applications of AdS/CFT to obtain fresh insight on the intricate dynamics of the QGP. Recent applications of holography involve far-from-equilibrium dynamics, where one studies 
how a non-equilibrium initial state evolves towards equilibrium using numerical general relativity and the holographic dictionary to compute the stress-energy tensor $\left\langle T_{\mu \nu}\right\rangle$ [5]. In the context of the boost-invariant Bjorken flow [11], it is possible to construct its holographic version to study how the system approaches equilibrium [12], and here we use the term hydrodynamization time to refer to the instant where a hydrodynamic solution matches the full non-linear holographic solution [13].

Inspired by these facts, here we study the hydrodynamization process of a strongly coupled plasma in the vicinity of a critical point, i.e., we want to assess the effects that a critical point has on the hydrodynamization time [14]. For such a task, we employ a conformal top-down holographic model dubbed as 1-R charged black hole (1RCBH) model [15], which is the holographic dual of a $\mathcal{N}=4 \mathrm{SYM}$ plasma at finite temperature and chemical potential featuring a critical point in its phase diagram.

In this work we use a mostly plus metric signature and natural units $\hbar=c=k_{B}=1$.

\section{Results}

The five-dimensional bulk gravitational Lagrangian that characterizes the $1 \mathrm{RCBH}$ model is

$$
2 \kappa_{5}^{2} \mathcal{L}=R-\frac{\left(\partial_{\mu} \phi\right)^{2}}{2}-V(\phi)-\frac{f(\phi)\left(F_{\mu v}\right)^{2}}{4},
$$

where $\kappa_{5}^{2}=8 \pi G_{5}, \phi$ is the dilaton field, $V(\phi)=-8 e^{\frac{\phi}{\sqrt{6}}}-4 e^{-\sqrt{\frac{2}{3}} \phi}$ (the AdS radius is set to unity), $F_{\mu \nu}=\partial_{[\mu} A_{v]}$ is the Maxwell field responsible for the chemical potential of the theory, and $f(\phi)=$ $e^{-2 \sqrt{\frac{2}{3}} \phi}$ is the coupling function between $\phi$ and $A_{\mu}$.

In order to describe the holographic Bjorken flow, we assume the following Ansatz for the metric and bulk fields

$$
\begin{aligned}
& d s^{2}=2 d \tau[d r-A(\tau, r) d \tau]+\Sigma(\tau, r)^{2}\left[e^{-2 B(\tau, r)} d \xi^{2}+e^{B(\tau, r)}\left(d x^{2}+d y^{2}\right)\right], \\
& \phi=\phi(\tau, r), \quad A=\Phi(\tau, r) d \tau,
\end{aligned}
$$

where $\tau$ is the proper time, and $r$ is the radial AdS coordinate with boundary at $r \rightarrow \infty$. The unkown functions $\{A, \Sigma, B\}$ of the metric that gives us information about the gravitational background are determined once the Einstein's equations for the Lagrangian (1) are solved. The field $\Sigma$ is related to the chemical potential $(\mu)$ when the system is in a local equilibrium state, i.e., $\Phi(r \rightarrow \infty)=\mu$. Moreover, since the flux is boost-invariant, the elements of the metric above do not depend on the rapidity $\xi$ [12].

With Equation (2) at hand, and given an initial condition for the bulk fields, one has to solve the time dependent Einstein's equations supplied by the dilaton and Maxwell equations to determine the time evolution of the holographic Bjorken flow. Evidently, to extract meaningful quantities, we need to compute the one-point functions $\left\langle T_{\mu \nu}\right\rangle,\left\langle J_{v}\right\rangle$, and $\left\langle\mathcal{O}_{\phi}\right\rangle$. The derivation of such formulas follow from the holographic renormalization procedure, which can be found in detail in Ref. [16].

Once the simulations are done, and the one-point functions are computed, one is able to see what the effect of the critical point on the hydrodynamization time is. In practice, to compute the hydrodynamization time $w_{\text {hydro }}(\mu / T)$, we compute the pressure anisotropy $\left(\Delta p=\left\langle T_{x}^{x}\right\rangle-\left\langle T_{\xi}^{\xi}\right\rangle\right)$ normalized by the energy density $\left(\varepsilon=\left\langle T_{\tau \tau}\right\rangle\right)$, i.e., $\left(\frac{\Delta p}{\varepsilon}\right)_{\text {numerical }}$ and then define this time as the instant where the inequality below holds

$$
\left|\left(\frac{\Delta p}{\varepsilon}\right)_{\text {numerical }}-\left(\frac{\Delta p}{\varepsilon}\right)_{\text {hydro }}\right| \leq 0.01\left(\frac{\Delta p}{\varepsilon}\right)_{\text {hydro }},
$$

where $\left(\frac{\Delta p}{\varepsilon}\right)_{\text {hydro }}$ refers to the Navier-Stokes solution [14]. Moreover, it is important to keep in mind that the measure of time here is defined by the dimensionless variable $w \equiv \hat{\varepsilon}^{1 / 4} \tau$, where variables with hat are defined as $\hat{X}=\kappa_{5}^{2} X$. We checked that varying the tolerance from 0.01 to 0.1 the qualitative result does not change. 
In Figure 1 one can see the holographic results represented by solid lines, while dashed lines are the Navier-Stokes solutions. The top-left plot shows $\Delta p$ and one can see that increasing the chemical potential tends to enhance the thermalization time. Indeed, to probe the effect of the chemical potential and the critical point, we have defined the subtracted hydrodynamization time

$$
\Delta w_{\text {hydro }} \equiv \frac{w_{\text {hydro }}(\mu / T)-w_{\text {hydro }}(0)}{w_{\text {hydro }}(0)} .
$$

The bottom-right of Figure 1 shows that the critical point has very pronounced effect on the hydrodynamization time, i.e., it takes longer for the plasma to acquire hydrodynamic behavior in the vicinity of the critical point.
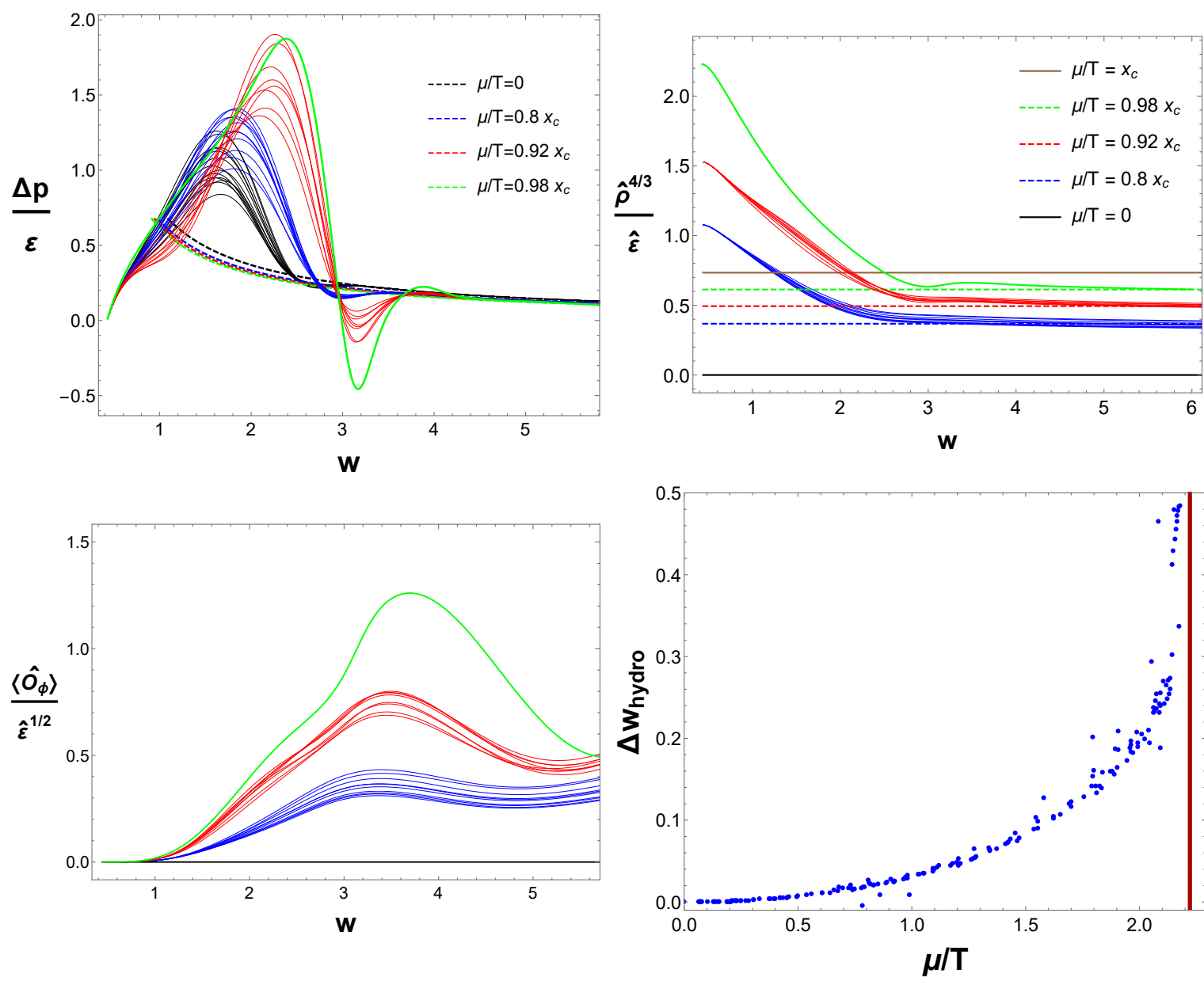

Figure 1. Results for the holographic Bjorken flow evolution on top of 1 RCBH backgrounds $\left(x_{c} \equiv\right.$ $(\mu / T)_{c}=\pi / \sqrt{2}$ is the critical point [16]). Top Left: Pressure anisotropy. Top Right: Charge density. Bottom Left: Time evolution. Bottom Right: Subtracted hydrodynamization time (4) as function of the chemical potential. The red line indicates the critical point.

\section{Conclusions}

The AdS/CFT duality provides a powerful method to explore far-from-equilibrium dynamics of strongly coupled plasmas. Our analysis of the $1 \mathrm{RCBH}$ model revealed a substantial delay of the hydrodynamization time as one approaches critical point. Evidently, it is not known if this also happens in QCD but other results based on the Functional Renormalization Group (FRG) suggest that this is indeed the case [17]. We intend to extend these far-from-equilibrium calculations to consider more realistic bottom-up models [18] undergoing flows that have more features than those displayed by the Bjorken flow [19]. 
Funding: R.C. acknowledges financial support by the São Paulo Research Foundation (FAPESP) under FAPESP grant number 2016/09263-2.

Acknowledgments: The author thanks Romulo Rougemont and Jorge Noronha for collaboration and comments on this manuscript.

\section{References}

1. Gyulassy, M.; McLerran, L. New forms of QCD matter discovered at RHIC. Nucl. Phys. A 2005, 750, 30. doi:10.1016/j.nuclphysa.2004.10.034.

2. Heinz, U.; Snellings, R., Collective flow and viscosity in relativistic heavy-ion collisions. Ann. Rev. Nucl. Part. Sci. 2013, 63, 123. doi:10.1146/annurev-nucl-102212-170540.

3. Meyer, H.B., Transport Properties of the Quark-Gluon Plasma: A Lattice QCD Perspective. Eur. Phys. J. A 2011, 47, 86. doi:10.1140/epja/i2011-11086-3.

4. Maldacena, J.M., The Large N limit of superconformal field theories and supergravity. Int. J. Theor. Phys. 1999, 38, 1113. doi:10.1023/A:1026654312961.

5. Chesler, P.M.; Yaffe, L.G., Numerical solution of gravitational dynamics in asymptotically anti-de Sitter spacetimes. JHEP 2014, 1407, 086. doi:10.1007/JHEP07(2014)086.

6. Alford, M.G.; Schmitt, A.; Rajagopal, K.; Schäfer, T., Color superconductivity in dense quark matter. Rev. Mod. Phys. 2008, 80, 1455. doi:10.1103/RevModPhys.80.1455.

7. Philipsen, O. The QCD equation of state from the lattice. Prog. Part. Nucl. Phys. 2013, 70, 55. doi:10.1016/j.ppnp.2012.09.003.

8. Bazavov, A., The QCD Equation of State to $\mathcal{O}\left(\mu_{B}^{6}\right)$ from Lattice QCD. Phys. Rev. D 2017, 95, 054504. doi:10.1103/PhysRevD.95.054504.

9. DeWolfe, O.; Gubser, S.S.; Rosen, C., Dynamic critical phenomena at a holographic critical point. Phys. Rev. D 2011, 84, 126014. doi:10.1103/PhysRevD.84.126014.

10. Kovtun, P.; Son, D.T.; Starinets, A.O., Viscosity in strongly interacting quantum field theories from black hole physics. Phys. Rev. Lett. 2005, 94, 111601. doi:10.1103/PhysRevLett.94.111601.

11. Bjorken, J.D., Highly Relativistic Nucleus-Nucleus Collisions: The Central Rapidity Region. Phys. Rev. D 1983, 27, 140. doi:10.1103/PhysRevD.27.140 .

12. Chesler, P.M.; Yaffe, L.G., Boost invariant flow, black hole formation, and far-from-equilibrium dynamics in N = 4 supersymmetric Yang-Mills theory. Phys. Rev. D 2010, 82, 026006. doi:10.1103/PhysRevD.82.026006.

13. Heller, M.P.; Janik, R.A.; Witaszczyk, P., The characteristics of thermalization of boost-invariant plasma from holography. Phys. Rev. Lett. 2012, 108, 201602. doi:10.1103/PhysRevLett.108.201602.

14. Critelli, R.; Rougemont, R.; Noronha, J., Holographic Bjorken flow of a hot and dense fluid in the vicinity of a critical point. Phys. Rev. D 2019, 99, 066004. doi10.1103/PhysRevD.99.066004.

15. Gubser, S.S., Thermodynamics of spinning D3-branes. Nucl. Phys. B 1999, 551, 667. doi:10.1016/S0550-3213(99)00194-7.

16. Critelli, R.; Rougemont, R.; Noronha, J., Homogeneous isotropization and equilibration of a strongly coupled plasma with a critical point. JHEP 2017, 1712, 029. doi:10.1007/JHEP12(2017)029.

17. Bluhm, M.; Jiang, Y.; Nahrgang, M.; Pawlowski, J.M.; Rennecke, F.; Wink, N., Time-evolution of fluctuations as signal of the phase transition dynamics in a QCD-assisted transport approach. Nucl. Phys. A 2019, 982, 871. doi:10.1016/j.nuclphysa.2018.09.058.

18. Critelli, R.; Noronha, J.; Noronha-Hostler, J.; Portillo, I.; Ratti, C.; Rougemont, R., Critical point in the phase diagram of primordial quark-gluon matter from black hole physics. Phys. Rev. D 2017, 96, 096026. doi:10.1103/PhysRevD.96.096026.

19. Gubser, S.S., Symmetry constraints on generalizations of Bjorken flow. Phys. Rev. D 2010, 82, 085027. doi:10.1103/PhysRevD.82.085027.

(C) 2019 by the authors. Licensee MDPI, Basel, Switzerland. This article is an open access article distributed under the terms and conditions of the Creative Commons Attribution (CC BY) license (http://creativecommons.org/licenses/by/4.0/). 